\title{
An MMSE Strategy at Relays With Partial CSI for a Multi-Layer Relay Network
}

\author{
Pannir Selvam Elamvazhuthi, Student Member, IEEE, Bikash Kumar Dey, Member, IEEE, and \\ Srikrishna Bhashyam, Senior Member, IEEE
}

\begin{abstract}
We consider a relay network with a single source-destination pair and multiple layers of relays between them. We assume that these layers sequentially relay the signal transmitted by the source to the destination. Unlike existing work, we also assume that the destination and all the forward layers present between the transmitting layer and the destination receive signals during every transmission phase. We optimally combine these signals, say $\mu$ of them, using a precoder at each relay layer for onward transmission. We obtain this precoding matrix by minimizing the meansquared error (MSE) at the relays, and do not require channelstate-information (CSI) of the forward channels at the relays unlike existing systems that minimize MSE at the destination and require CSI of the forward channels at the relays. Our closed-form solution for this matrix is valid for any $K$ number of layers, whereas minimizing MSE at the destination does not have closed-form solution for $K>1$. For $K>1$, we enhance an existing scheme to obtain a sub-optimal closed-form precoder solution and use it for comparison. We show using simulations that our scheme approaches the bit-error-rate (BER) performance of this scheme, when $\mu$ is increased, even with partial CSI.
\end{abstract}

Index Terms-MMSE, channel-state-information, multi-layer relay network, relay precoder, amplify-and-forward.

\section{INTRODUCTION}

$\mathbf{J}$ ING and Hassibi [1] proved that a spatially distributed network of single-antenna radio nodes can emulate a multiple-input multiple-output (MIMO) communication system. They showed that this creates a distributed space-time code and achieves the same diversity as that of a MIMO system at high total transmitted power. These authors also extended it to include multiple-antenna nodes in [2] and [3].

When multiple radio nodes are present, the effectiveness of cooperative communication [4], [5] can be increased by

Manuscript received March 17, 2013; revised August 05, 2013; accepted September 24, 2013. Date of publication October 04, 2013; date of current version December 23, 2013. The associate editor coordinating the review of this manuscript and approving it for publication was Prof. Samson Lasaulce. The work of P. Selvam and B. K. Dey was supported in part by Bharti Centre for Communication.

P. S. Elamvazhuthi is with Delphi Automotive Systems Pvt. Ltd., Technical Center India, Kalyani Platina, Whitefield, Bengaluru 560066, India (e-mail: pannir.selvam@delphi.com).

B. K. Dey is with the Indian Institute of Technology Bombay, Powai, Mumbai 400076, India (e-mail: bikash@ee.iitb.ac.in).

$\mathrm{S}$. Bhashyam is with the Indian Institute of Technology Madras, Chennai 600036, India (e-mail: skrishna@ee.iitm.ac.in).

Color versions of one or more of the figures in this paper are available online at $\mathrm{http}: / /$ ieeexplore.ieee.org.

Digital Object Identifier 10.1109/TSP.2013.2284471 arranging these nodes into a layered architecture to relay information. Pottie and Kaiser [6] showed how a distributed and layered signal processing architecture can overcome the energy and bandwidth constraints in many wireless sensor network applications.

A relay can use a simple forwarding technique called amplify-and-forward (AF) [7], in which it amplifies what is received and transmits. Other well-known relaying methods include decode-and-forward (DF) (e.g., [8]), coded cooperation (e.g., [9]), compress-and-forward (e.g., [10], [11]), and partial-decode-and-forward (PDF) (e.g., [12]). Chiu et al. [13] designed the precoder for not only the relay but also the source, while using the PDF strategy at the relays. They used orthogonal space-time block coding [14] in both the source and the relay transmissions. Though other relaying protocols can perform better, the AF protocol is widely considered interesting because of its simplicity of implementation. In this paper, we restrict our attention to the AF protocol. In an AF multi-layer relay system, the performance of the system depends on the precoders used at the relays, and we consider the problem of designing such precoders.

Our system model consists of a set of single-antenna radio nodes grouped into $K$ layers of relays, $\mathrm{L}_{k}, k \in[1, K]$, between a source and a destination (we will call the source $\mathrm{S}$ or $\mathrm{L}_{0}$ and the destination $\mathrm{D}$ or $\mathrm{L}_{K+1}$ in the sequel) as shown in Fig. 1. Earlier work involving multiple layers of relays use only the signal reaching at a particular layer $\mathrm{L}_{k}$ from the preceding layer $\mathrm{L}_{k-1}$ to construct the signals transmitted from $\mathrm{L}_{k}$. On the contrary, in this paper, we construct the transmit signals at $\mathrm{L}_{k}$ using signals that have reached from $\mathrm{L}_{j}, j \in[0, k-1]$, although the signal from $\mathrm{L}_{j}, j \in[0, k-2]$ reaches $\mathrm{L}_{k}$ with lower power compared to that from $\mathrm{L}_{k-1}$. Thus, we take advantage of the broadcast nature of the wireless medium by utilizing overheard signals. We call these low power overheard signals as leaked signals.

We will call the channel states of the channels from $\mathrm{L}_{k-j}$ to $\mathrm{L}_{k}$, available at $\mathrm{L}_{k}$, to be backward CSI and the channel states of the channels from $\mathrm{L}_{k}$ to $\mathrm{L}_{k+j}$ as forward CSI. Here $j>$ 0 . Forward and backward CSI are together called the global CSI, and just the backward CSI is referred to as partial CSI. We note here that obtaining forward CSI requires either sending the estimated channels from the receiving nodes over reliable feedback channels, or direct estimation of these channels from the backward transmission (in a time-division duplex system). The feasibility of this depends on the application scenario.

Ding et al. [15] employed AF strategy at the relays, designed a unitary precoder, and achieved maximum diversity gain. The closed form expression of the precoder was extended to 


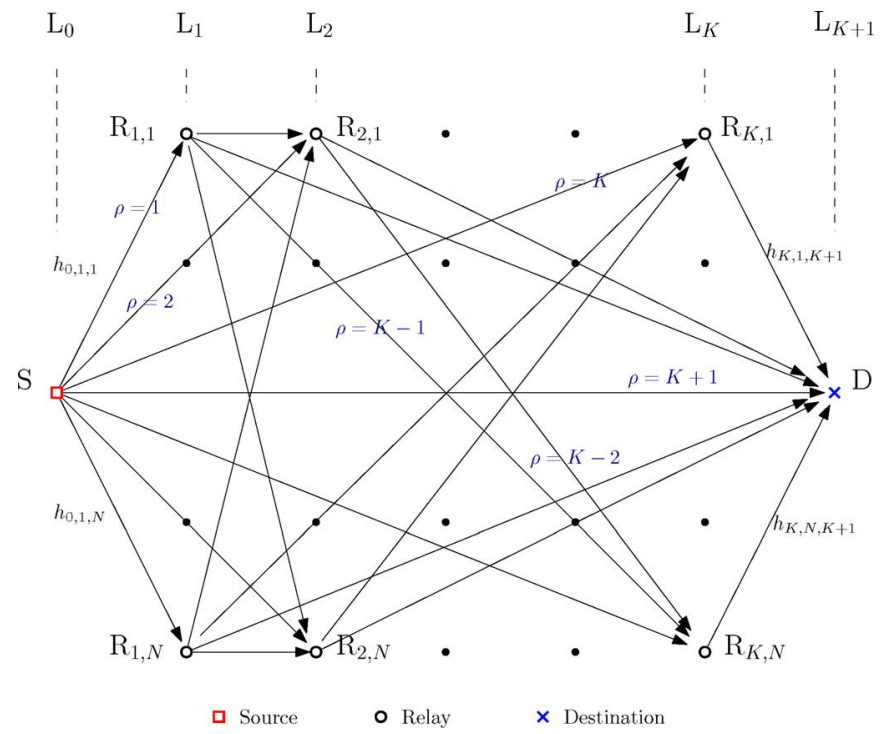

Fig. 1. A multi-layer relay network. Here $\mathrm{S}, \mathrm{L}_{k}, \mathrm{R}_{k, i}$, $\mathrm{D}$, and $\rho$ represent the source, $k$ th layer, $i$ th relay in $\mathrm{L}_{k}$, the destination, and the length of the corresponding link respectively. The relays are assumed to be half-duplex.

$M$-ary signals of larger constellation size in [16]. Gomadam and Jafar [17] obtained relay precoders by maximizing the receive signal-to-noise ratio (SNR) at the destination. Many authors [18]-[23] have considered minimizing the MSE at the destination (MMSED) to obtain relay precoders using global CSI. This global optimization is challenging, and authors of [17] and [23] considered two layers of relays and obtained the precoder by iterative techniques, while the authors of [18]-[22] derived the optimal precoders in closed-form for a single layer of relays. The disadvantage in an iterative technique is that at any iteration, only an approximate solution is found. For real-time computation at the relays, the quality of the solution then depends on the processing speed of the relays. Further, to the best of our knowledge, iterative solutions are also available only for upto two layers of relays, and their generalization to more layers or incorporating leaked signals is challenging under the MMSED criterion.

In some systems, the relays may be able to exchange information amongst themselves before transmission. In such a system, the relays are said to be cooperative. Otherwise, the precoder matrix would be diagonal and the relays are said to be non- $\mathrm{Co}-$ operative. All the literature discussed in the previous paragraph, showed the efficacy of the derived precoders when the relays are non-cooperative except [19], in which the authors derived the precoders for cooperative relays. For either cooperative and non-cooperative relays, to our knowledge, existing literature provides minimum MSE (MMSE) design of AF precoders:

- in closed form only when there is a single layer of relays, and in the form of iterative numerical solution when there are two layers,

- assuming that global CSI is available with the relays, and

- without considering leaked signals.

All the above concerns are addressed in this paper. Unlike [18]-[23], which adopted MMSED, we minimize MSE at the relays (MMSER) and obtain relay precoder matrices. We show that the MMSER strategy makes the optimal precoder design a layer-wise optimization as opposed to a global optimization. This yields optimal precoders in closed form for arbitrary number of relay layers even when leaked signals are used, and it requires partial CSI, i.e., only the backward CSI. In the absence of optimum MMSED precoder solution for more than two layers of relays, we have proposed some suboptimal MMSED precoding schemes, and we show using simulation that despite the lack of forward CSI, our MMSER precoding strategy outperforms/approaches the performance of these MMSED strategies by using more leaked signals.

\section{A. Contribution}

Our contributions in this paper are:

- For the multi-layer relay network shown in Fig. 1, we propose a novel MMSER relaying strategy, which does not require forward CSI in the transmitting nodes.

- We obtain closed form solutions for the optimum MMSER relay precoders for both cooperative and non-cooperative relays for arbitrary number of layers of relays while also including leaked signals.

- We enhance the MMSED strategies (though these enhancements may not be optimal) proposed in [18], [19], and [21] to work in this multi-layer network for meaningful comparison with MMSER.

- We show using simulations that combining more number of leaked signals improves the performance of MMSER, which outperforms/approaches that of MMSED schemes that use global CSI.

\section{B. Notation and Organization}

$\mathbf{I}_{N}$ denotes the $N \times N$ identity matrix and $\mathbf{i}_{N}$ is the vector of $N$ elements $[1,1, \ldots, 1]^{T}$. For $x \in \mathbb{Z},[x]^{+}$denotes zero if $x \leq$ 0 and $x$ if $x>0 . \xi \sim \mathcal{N}_{\mathbb{C}}\left(0, \sigma^{2}\right)$ represents a circularly symmetric complex Gaussian random variable with real and imaginary parts having mean 0 and variance $\sigma^{2} / 2 \cdot \operatorname{diag}\left[x_{1}, \ldots, x_{N}\right]$ is a diagonal matrix with diagonal elements $x_{i}, i \in[1, N]$.

The remainder of this paper is organized as follows. In Section II, we state the problem and introduce the MMSER strategy. Thereafter, in Section III, the MMSER strategy is presented in detail and the precoders for the relay layers are derived. Section IV gives details on how we extend and enhance the MMSED strategy, so that the BER performance of MMSER can be meaningfully compared. In Section $\mathrm{V}$, we describe the equalizer that is used at destination D for MMSER and MMSED schemes to decode the received vector. In Section VI, we present simulation results. Finally, in Section VII, we summarize and conclude the paper.

\section{System Model AND the Proposed Scheme}

In our system model shown in Fig. $1, \mathrm{R}_{i, m}$ denotes the $m$ th relay in the $i$ th layer and we assume that it is half-duplex, $\forall i, m$. We use $\ell$ and $h$ to represent the links and the channel coefficients respectively, with the first two subscripts denoting the transmitter and the next two the receiver. Therefore, the channel coefficients of the links, $\ell_{0, i, m}$ from $\mathrm{S} \rightarrow \mathrm{R}_{i, m}$, $\ell_{i, m, j, n}$ from $\mathrm{R}_{i, m} \rightarrow \mathrm{R}_{j, n}$, and $\ell_{j, n, K+1}$ from $\mathrm{R}_{j, n} \rightarrow \mathrm{D}$ are denoted as $h_{0, i, m}, h_{i, m, j, n}$, and $h_{j, n, K+1}$ respectively. Let $\mathbf{h}_{0, i} \in \mathbb{C}^{N \times 1}, \mathbf{H}_{i, j} \in \mathbb{C}^{N \times N}$, and $\mathbf{h}_{j, K+1} \in \mathbb{C}^{1 \times N}$ represent 
TABLE I

RECEIVED AND TRANSMITTED VeCTORS-RELAY LAYERS

\begin{tabular}{|c|c|c|}
\hline Layer & Received vector & Transmitted vector \\
\hline \hline $\mathrm{L}_{k}$, & $\mathbf{r}_{k}^{(0)}=\mathbf{h}_{0, k} t_{0}+\mathbf{u}_{k}^{(0)}$, & $\mathbf{t}_{k}=\mathbf{F}_{k} \mathbf{r}_{k}$, where \\
$1 \leq k$ & $\mathbf{r}_{k}^{(i)}=\mathbf{H}_{i, k} \mathbf{t}_{i}+\mathbf{u}_{k}^{(i)}$ & $\mathbf{r}_{k}^{T}=\left[\mathbf{r}_{k}^{(n) T}, \cdots, \mathbf{r}_{k}^{(k-1) T}\right]$ \\
$\leq K$ & $\forall i \in[n, k-1]$ and $i \neq 0$. & with $n=[k-\mu]^{+}$. \\
\hline
\end{tabular}

the vectors/matrices of channel coefficients from $\mathrm{S}$ to $\mathrm{L}_{i}, \mathrm{~L}_{i}$ to $\mathrm{L}_{j}$, and $\mathrm{L}_{j}$ to $\mathrm{D}$ respectively. We assume that the channels are Rayleigh fading and quasi static.

Let $\mathbb{L}$ be the set of all links and $\mathbb{L}_{i, j} \subset \mathbb{L}, i<j$ be the set of links from $\mathrm{L}_{i}$ to $\mathrm{L}_{j}$. As an example, for $i \in[1, K-1], j \in$ $[i+1, K]$, the link set $\mathbb{L}_{i, j}$ is given by

$$
\mathbb{L}_{i, j}=\left\{\ell_{i, 1, j, 1}, \ldots, \ell_{i, N, j, N}\right\} .
$$

Let us define the length of the link $\ell_{i, m, j, n}$ as $\rho_{i j} \triangleq j-i$, or simply by $\rho$ when the layers are understood from the context. All the links in link set $\mathbb{L}_{i, j}$ have the same length $\rho_{i j}=j-i$.

Now, let $\mathscr{L}_{\rho}$ be a class of subsets $\mathbb{L}_{i, j}$ of $\mathbb{L}$ with length $\leq \rho$. Clearly, $\mathscr{L}_{i} \subset \mathscr{L}_{j}$, if $i<j$. As an example, link class $\mathscr{L}_{2}$ is given by

$$
\mathscr{L}_{2}=\left\{\mathbb{L}_{0,1}, \mathbb{L}_{0,2}, \mathbb{L}_{1,2}, \mathbb{L}_{1,3}, \ldots, \mathbb{L}_{K-1, K+1}, \mathbb{L}_{K, K+1}\right\} .
$$

\section{The Proposed MMSER- $\mu$ Strategy}

Let us consider the $K+1$ hop network shown in Fig. 1. In the MMSER- $\mu(\mu \in[1, K+1])$ strategy, $\mathrm{S}$ transmits in phase 0 , and $\mathrm{L}_{1}, \mathrm{~L}_{2}, \ldots, \mathrm{L}_{\mu}$ receive and store for later use; $\mathrm{L}_{1}$ transmits in phase 1 , and $\mathrm{L}_{2}, \ldots, \mathrm{L}_{\mu+1}$ receive and store for later use and so on till phase $K$, when $\mathrm{D}$ receives from $\mathrm{L}_{K}$. To be specific, in MMSER- $\mu$, the relays and D store all signals received through the link sets in $\mathscr{L}_{\mu}$ for later use. For $\mu_{1}<\mu_{2}$, MMSER- $\mu_{2}$ would use more number of leaked signals, and thus is expected to perform better than MMSER- $\mu_{1}$.

We assume synchronous reception and transmission at the relay nodes and all noise signals added at the receiver front-ends are complex zero-mean independent and identically distributed (i.i.d.) Gaussian random variables with variance $\sigma_{u}^{2}$.

Let us denote the transmitted, received, and noise signals by $t, r$, and $u$ respectively with subscript and superscript on them denoting layer and phase respectively. Let the signal transmitted by $\mathrm{S}$ be $t_{0}=\sqrt{p_{0}} s$ with an average power of $p_{0}$ Watts, where $s \in \mathbb{C}$ is a unit variance constellation point. In various phases, the layer $\mathrm{L}_{k}$ would have received $k-n$ vectors each of size $N$ from previous transmissions, starting from phase $n$ till phase $k-1$, where $n=[k-\mu]^{+}$. All these vectors are stacked together as given in Table I to form the overall received vector $\mathbf{r}_{k} \in$ $\mathbb{C}^{(k-n) N \times 1}$.

For example, let us take $K=8$ and $\mu=3$. Here, $n=$ $[k-3]^{+}=0$ for $k=2$ and $n=[k-3]^{+}=4$ for $k=7$. Hence, $\mathrm{L}_{2}$ and $\mathrm{L}_{7}$ would have the overall received vectors

$$
\mathbf{r}_{2}=\left[\begin{array}{l}
\mathbf{r}_{2}^{(0)} \\
\mathbf{r}_{2}^{(1)}
\end{array}\right] \text { and } \mathbf{r}_{7}=\left[\begin{array}{l}
\mathbf{r}_{7}^{(4)} \\
\mathbf{r}_{7}^{(5)} \\
\mathbf{r}_{7}^{(6)}
\end{array}\right]
$$

respectively. Also $\mathbf{r}_{2} \in \mathbb{C}^{2 N \times 1}$ and $\mathbf{r}_{7} \in \mathbb{C}^{3 N \times 1}$.

The stacked received vector is transmitted by $\mathrm{L}_{k}$ relays, after precoding with $\mathbf{F}_{k}$ in phase $k$ as shown in Table I. The precoder matrix $\mathbf{F}_{k} \in \mathbb{C}^{N \times(k-n) N}$ at $\mathrm{L}_{k}$ is given by

$$
\mathbf{F}_{k}=\left[\mathbf{F}_{k, n}, \ldots, \mathbf{F}_{k, k-1}\right],
$$

where $\mathbf{F}_{k, i} \in \mathbb{C}^{N \times N}$ with $n \leq i \leq k-1$ and $n=[k-\mu]^{+}$. The precoder submatrices $\mathbf{F}_{k, i}$ can be selected to be non-diagonal or diagonal depending upon whether the relays would cooperate or not respectively.

In phases $i=K-\mu+1$ to $i=K$, D receives $r_{K+1}^{(i)}=$ $\mathbf{h}_{i, K+1} \mathbf{t}_{i}+u_{K+1}^{(i)}$. If $K-\mu+1=0$, then it receives $r_{K+1}^{(0)}=$ $h_{0, K+1} t_{0}+u_{K+1}^{(0)}$ from $\mathrm{S}$ directly in phase 0 .

Now, we define the cost function at the relay layer $\mathrm{L}_{k}$ to be the MSE

$$
J_{k} \triangleq E\left[\left\|\mathbf{s}-\mathbf{t}_{k}\right\|^{2}\right],
$$

where $\mathbf{s}=[s, \ldots, s]^{T} \in \mathbb{C}^{N \times 1}, \mathbf{t}_{k}=\mathbf{F}_{k} \mathbf{r}_{k}$ as given in Table I and $E[\cdot]$ is the expectation operator. The relays then transmit a scaled version of the solution to meet the layer-wise power constraint. The cost function given in (2) is motivated by the following facts:

- Somewhat similar to the principle behind regenerative relaying (DF), the relays are desired to transmit a signal that is close to the source symbol or its scaled version.

- The otherwise complex optimization problem (with MMSED criterion) is replaced by a smaller layer-wise optimization problem which, as we will see, yields a solution in closed form.

- The requirement of only backward CSI gives an added practical advantage.

Now, our aim is to minimize $J_{k}$ under the power constraint $E\left[\mathbf{t}_{k}^{H} \mathbf{t}_{k}\right] \leq p_{k}$ and find the precoder matrices $\mathbf{F}_{k}, \forall k \in[1, K]$.

\section{MMSER PRECODER}

Khajehnouri and Sayed [18] minimized the MSE

$$
J_{D}=E\left[\left|s-\mathbf{h}_{1,2} \mathbf{t}_{1}\right|^{2}\right]
$$

at the destination and found the precoder matrix for $\mathrm{L}_{1}$, when $K=1$ with no power constraint. Here, $\mathbf{h}_{1,2} \in \mathbb{C}^{1 \times N}$ and $\mathbf{t}_{1} \in \mathbb{C}^{N \times 1}$. Krishna et al. [19] derived a non-diagonal precoder matrix for cooperative relays with average power constraint $p_{1}$. To compare with their results, these authors modified the $i$ th diagonal element of the precoder matrix to restrain power in the Khajehnouri-Sayed scheme [18] as

$$
f_{1, i}=\frac{p_{1}^{\frac{1}{2}} h_{0,1, i}^{*} h_{1, i, 2}^{*}}{\left|h_{1, i, 2}\right|^{2}\left[\sum_{j=1}^{N} \frac{\left|h_{0,1, j}\right|^{2}}{\mid h_{1, j, 2}}\left(p_{0}\left|h_{0,1, j}\right|^{2}+\sigma_{u}^{2}\right)\right]^{\frac{1}{2}}}
$$

for non-cooperative relays in Khajehnouri-Sayed equation (24). Lee et al. [20] obtained $f_{1, i}$ using constrained optimization [24] for non-cooperative relays as

$$
f_{1, i}=\frac{p_{1}^{\frac{1}{2}} h_{0,1, i}^{*} h_{1, i, 2}^{*}}{\left[\sum_{j=1}^{N}\left|h_{0,1, j}\right|^{2}\left|h_{1, j, 2}\right|^{2}\left(p_{0}\left|h_{0,1, j}\right|^{2}+\sigma_{u}^{2}\right)\right]^{\frac{1}{2}}},
$$


where we have removed the uncertainty channel terms to match the scope of this paper. In both (3) and (4), we have changed symbol notation to be consistent with this paper.

Let us call these non-cooperative systems, which use (3) and (4), as MMSED-Khajehnouri/Krishna (MMSED-KK) and MMSED-Lee (MMSED-L) respectively. We also call the cooperative system proposed by Krishna et al. [19] as MMSED-Krishna (MMSED-K). We note that, both MMSED-KK and MMSED-L require $h_{1, i, 2}$ or forward CSI at the relay $\mathrm{R}_{1, i}, i \in[1, N]$ from (3) and (4) respectively.

In our strategy, we minimize the MSE at the relays resulting in precoders that do not depend on forward CSI, and use leaked signals to improve the performance. Let us now derive the precoders for MMSER- $\mu$ for any $K$.

\section{A. MMSER Precoder Matrix at $L_{k}, k \in[1, K]$}

In our proposed MMSER scheme, each layer obtains an estimate of the signal vector $\mathbf{s}$. This estimate or a scaled version of this estimate can be transmitted, subject to the sum transmit power constraint for each layer of relays. Expanding the expression of the MSE given in (2), we get

$$
J_{k}\left(\mathbf{F}_{k}\right)=E\left[\left(\mathbf{s}-\mathbf{F}_{k} \mathbf{r}_{k}\right)^{H}\left(\mathbf{s}-\mathbf{F}_{k} \mathbf{r}_{k}\right)\right] .
$$

Now, the estimate is obtained by finding the optimum $\mathbf{F}_{k}$ given by $\hat{\mathbf{F}}_{k}=\arg \min _{\mathbf{F}_{k}} J_{k}\left(\mathbf{F}_{k}\right)$, subject to the constraint $E\left[\mathbf{t}_{k}^{H} \mathbf{t}_{k}\right] \leq p_{k}$. We write the constraint function as

$$
C_{k}\left(\mathbf{F}_{k}\right)=E\left[\mathbf{t}_{k}^{H} \mathbf{t}_{k}\right]-p_{k} \leq 0
$$

and let $\mathcal{D}=\operatorname{domain}\left(\mathbf{F}_{k}\right) \bigcap$ domain $\left(C_{k}\right)$. This problem is an MMSE estimation problem with a convex constraint on the estimate. It is a convex optimization problem with a unique solution as discussed in [25].

Now, given the optimization variable $\mathbf{F}_{k} \in \mathbb{C}^{N \times(n-k) N}$, the cost function $J_{k}: \mathbb{C}^{N \times(n-k) N} \rightarrow \mathbb{R}$, and the inequality constraint function $C_{k}: \mathbb{C}^{N \times(n-k) N} \rightarrow \mathbb{R}$, we define the Lagrangian $\mathcal{L}_{k}: \mathbb{C}^{N \times(n-k) N} \times \mathbb{R} \rightarrow \mathbb{R}$ as

$$
\mathcal{L}_{k}\left(\mathbf{F}_{\mathbf{k}}, \lambda_{k}\right) \triangleq J_{k}\left(\mathbf{F}_{\mathbf{k}}\right)+\lambda_{k} C_{k}\left(\mathbf{F}_{k}\right),
$$

where $\lambda_{k} \geq 0$ is the Lagrange multiplier and the domain of $\mathcal{L}_{k}=\mathcal{D} \times \mathbb{R}$.

Claim 1: (1) When the relays cooperate, the optimum precoder matrix $\hat{\mathbf{F}}_{k}$ is given by

$$
\hat{\mathbf{F}}_{k}=\frac{p_{k}^{\frac{1}{2}}}{\left[\operatorname{Tr}\left[\mathbf{R}_{s r_{k}}^{H} \mathbf{R}_{s r_{k}} \mathbf{R}_{r_{k}}^{-1}\right]\right]^{\frac{1}{2}}} \mathbf{R}_{s r_{k}} \mathbf{R}_{r_{k}}^{-1},
$$

where

$$
\mathbf{R}_{s r_{k}}=E\left[\mathbf{s r}_{k}^{H}\right] \text { and } \mathbf{R}_{r_{k}}=E\left[\mathbf{r}_{k} \mathbf{r}_{k}^{H}\right] .
$$

(2) When the relays do not cooperate, the optimum precoder vector $\hat{\mathbf{f}}_{k, l}$ is given by

$$
\hat{\mathbf{f}}_{k, l}=\frac{p_{k}^{\frac{1}{2}}}{\left[\sum_{l=1}^{N} \operatorname{Tr}\left(\Upsilon_{k l}^{s}{ }^{H} \Upsilon_{k l}^{s} \Upsilon_{k l}^{-1}\right)\right]^{\frac{1}{2}}} \Upsilon_{k l}^{s} \Upsilon_{k l}^{-1},
$$

where

$$
\begin{aligned}
\mathbf{f}_{k, l} & =\left[f_{k, n, l}, \ldots, f_{k, k-1, l}\right], \\
\Upsilon_{k l}^{s} & =\left[\gamma_{k l}^{s(n)}, \ldots, \gamma_{k l}^{s(k-1)}\right],
\end{aligned}
$$

and

$$
\Upsilon_{k l}=\left[\begin{array}{ccc}
\gamma_{k l}^{(n)(n)} & \ldots & \gamma_{k l}^{(n)(k-1)} \\
\vdots & \ddots & \vdots \\
\gamma_{k l}^{(k-1)(n)} & \cdots & \gamma_{k l}^{(k-1)(k-1)}
\end{array}\right], \quad l \in[1, N]
$$

Here $f_{k, i, l}, \gamma_{k l}^{s(j)}$, and $\gamma_{k l}^{(i)(j)}$ represent the $l$ th diagonal elements of $\mathbf{F}_{k, i}, \mathbf{R}_{s r_{k}^{(j)}}=E\left[\mathbf{s r}_{k}^{(j) H}\right]$, and $\mathbf{R}_{r_{k}^{(i)} r_{k}^{(j)}}=E\left[\mathbf{r}_{k}^{(i)} \mathbf{r}_{k}^{(j) H}\right]$ respectively.

Proof: See Appendix A.

We notice the similarity of (8) and (9), where $\mathbf{R}_{s r_{k}}$ and $\mathbf{R}_{r_{k}}$ are analogous to $\Upsilon_{k l}^{s}$ and $\Upsilon_{k l}$ respectively, except for the extra summing operator in the denominator in (9).

For the non-cooperative case, the optimum precoder $\hat{\mathbf{F}}_{k}$, is made from the optimum vectors, $\mathbf{f}_{k, l}$, by noting that these vectors give the $l$ th diagonal elements of all submatrices, $\mathbf{F}_{k, i}, i \in$ $[n, k-1], n=[k-\mu]^{+}$, that make up the precoder.

The sum transmit power constraint at each layer allows for optimal allocation of power among the relays depending on the quality of the estimate at each relay. Specializing (8) to layer 1, we get

$$
\hat{\mathbf{F}}_{1}=\frac{p_{1}^{\frac{1}{2}}}{\left[N\left(p_{0}\left\|\mathbf{h}_{0,1}\right\|^{2}+\sigma_{u}^{2}\right)\right]^{\frac{1}{2}}} \frac{\mathbf{i}_{N} \mathbf{h}_{0,1}^{H}}{\left\|\mathbf{h}_{0,1}\right\|}
$$

as $\mathbf{R}_{s r_{1}}=p_{0}^{\frac{1}{2}} \mathbf{i}_{N} \mathbf{h}_{0,1}^{H}$ and $\mathbf{R}_{r_{1}}=p_{0} \mathbf{h}_{0,1} \mathbf{h}_{0,1}^{H}+\sigma_{u}^{2} \mathbf{I}_{N}$ from Table I. From (13), we can observe that the relays with better backward channels are allocated more power.

\section{B. Information Required for MMSER Precoders}

From (8) and (9), we can see that the precoders of MMSER depend on two correlation matrices $\mathbf{R}_{s r_{k}}$ and $\mathbf{R}_{r_{k}}$. From Table I, we see that, these matrices depend on $\mathbf{H}_{i, k}, \forall i \in[n, k-1], i \neq 0$ (if $i=0$, then on $\mathbf{h}_{0, k}$ ), and the precoder matrices $\hat{\mathbf{F}}_{i}$ for $i \in[n, k-1]$. Since $\hat{\mathbf{F}}_{1}$ (see (13)) depends only on backward CSI at $\mathrm{L}_{1}$, the information required to construct $\hat{\mathbf{F}}_{k}$ at $\mathrm{L}_{k}$ is also the backward CSI at $\mathrm{L}_{k}$. Therefore, MMSER- $\mu$ does not require forward CSI.

\section{EXTENSION OF MMSED SCHEMES}

Derivation of precoders for MMSED schemes, MMSED-KK and MMSED-L ((3) and (4) give the diagonal elements of the precoders of these schemes for $K=1$ ), is complicated when $K>1$. The system in [23] has two layers, i.e., $K=2$, and the precoders are obtained iteratively. In this Section, we enhance the MMSED strategies (though these enhancements may not be optimal) proposed in [18], [19], and [21] to obtain closed-form solutions to precoders in a multi-layer network for meaningful comparison with the MMSER- $\mu$ system proposed in this paper. We call these systems E-MMSED, for Enhanced-MMSED systems, and find their precoders to be dependent on global CSI as shown in (17) and (18). 


\section{A. E-MMSED Strategy}

For a meaningful comparison, we consider the total number of phases as $K+1$, and the total power transmitted as $P$ to be the same as that used by MMSER. We assume: $\mathrm{S}$ transmits $K_{0}$ times in as many phases; all the relays average their received signals and transmit $K_{1}=K-K_{0}+1$ times in as many phases. Thus, D would have a vector of $K_{1}$ received signals.

$\mathrm{S}$ transmits $t_{0}^{\prime}=\sqrt{p_{0}^{\prime}} s$ repeatedly $K_{0}$ times from phase zero to phase $K_{0}-1$, and the relays follow suit transmitting a signal vector $\mathbf{t}$, which is explained later, from phase $K_{0}$ to $K$. For each of these transmissions, when $\mathrm{S}$ transmits or the relays transmit, the channel does not vary as we assume a slow varying channel. Hence, the average power $p_{0}^{\prime}$ can be equally divided in various phases when $\mathrm{S}$ transmits and $p_{r}^{\prime}$ when the relays transmit. Thus, $p_{0}^{\prime}=p_{0} / K_{0}$ Watts and $p_{r}^{\prime}=p_{r} / K_{1}$ Watts respectively, with $p_{0}+p_{r}=P$, the total power available.

The relays in all the layers would receive in phase $k, k \in$ $\left[0, K_{0}-1\right]$, a vector $\mathbf{r}^{(k)} \in \mathbb{C}^{K N \times 1}$ given by

$$
\begin{array}{r}
\mathbf{r}^{(k)}=t_{0}^{\prime} \mathbf{h}_{0}+\mathbf{u}^{(k)}, k \in\left[0, K_{0}-1\right] \\
\text { where } \mathbf{h}_{0}=\left[\begin{array}{c}
\mathbf{h}_{0,1} \\
\vdots \\
\mathbf{h}_{0, K}
\end{array}\right] \text { and } \mathbf{u}^{(k)}=\left[\begin{array}{c}
\mathbf{u}_{1}^{(k)} \\
\vdots \\
\mathbf{u}_{K}^{(k)}
\end{array}\right]
\end{array}
$$

with $\mathbf{u}_{i}^{(k)}=\left[u_{i, 1}^{(k)} \ldots u_{i, N}^{(k)}\right]^{T}, i \in[1, K]$.

We assume that all the relays transmit together in their transmission phases, as though they are in a single layer. We also assume that the noise is uncorrelated, i.e.,

$$
E\left[\mathbf{u}^{(k)} \mathbf{u}^{(l) H}\right]=\sigma_{u}^{2} \delta(k-l) \mathbf{I}_{K N},
$$

where $\delta(m)=1$ when $m=0$ and $\delta(m)=0$ when $m \neq 0$ is the Kronecker delta function.

The relays average all the signals received and repeat transmission of the signal $\mathbf{t}=\mathbf{F r}_{\mathrm{av}}$, in phases $K_{0}$ to $K$, where

$$
\mathbf{r}_{\mathrm{av}}=\frac{1}{K_{0}} \sum_{i=0}^{K_{0}-1} \mathbf{r}^{(i)}=t_{0}^{\prime} \mathbf{h}_{0}+\frac{1}{K_{0}} \sum_{i=0}^{K_{0}-1} \mathbf{u}^{(i)}
$$

from (14). As $\mathbf{F} \in \mathbb{C}^{K N \times K N}$ is a diagonal precoder matrix, let us define it as $\mathbf{F} \triangleq \operatorname{diag}\left[f_{1,1}, \ldots, f_{1, N}, f_{2,1}, \ldots, f_{2, N}, \ldots, f_{K, 1}, \ldots, f_{K, N}\right]$, where $f_{i, m}$ is the multiplying factor of the relay $\mathrm{R}_{i, m}$.

From (15), $\mathbf{t}=\mathbf{F r}_{\mathrm{av}}$ becomes

$$
\mathbf{t}=t_{0}^{\prime} \mathbf{F} \mathbf{h}_{0}+\frac{\mathbf{F}}{K_{0}} \sum_{i=0}^{K_{0}-1} \mathbf{u}^{(i)}
$$

which is transmitted $K_{1}$ times, so that the total number of phases would be $K+1$, the same as that of MMSER.

Now, we will derive precoders for enhanced MMSED-KK (E-MMSED-KK) and enhanced MMSED-L (E-MMSED-L) schemes.

1) Precoder of E-MMSED-KK: We take (3) and replace $p_{0}$, the power transmitted by $\mathrm{S}$, with $p_{0} / K_{0}$ and $p_{1}$, the power transmitted by the relays, with $p_{r} / K_{1}$, as we allocate fractions of powers to them due to their multiple transmissions. Further, the noise variance $\sigma_{u}^{2}$ is replaced by $\sigma_{u}^{2} / K_{0}$, since the noise variance at each of the relays $\mathrm{R}_{i, m}, i \in[1, K], m \in[1, N]$ after averaging over the $K_{0}$ phases (in (15)) is $\sigma_{u}^{2} / K_{0}$. Therefore, we get $f_{i, m}, i \in[1, K]$ and $m \in[1, N]$, a diagonal element of the precoder of E-MMSED-KK as

$$
f_{i, m}=\frac{\left[\frac{p_{1} K_{0}}{K_{1}}\right]^{\frac{1}{2}} h_{0, i, m}^{*} h_{i, m, K+1}^{*} /\left|h_{i, m, K+1}\right|^{2}}{\left[\sum_{k=1}^{K} \sum_{l=1}^{N} \frac{\left|h_{0, k, l}\right|^{2}}{\left|h_{k, l, K+1}\right|^{2}}\left(p_{0}\left|h_{0, k, l}\right|^{2}+\sigma_{u}^{2}\right)\right]^{\frac{1}{2}}} .
$$

2) Precoder of E-MMSED-L: Similarly, to get the diagonal elements of the precoder of E-MMSED-L, we replace the signal power transmitted and noise variance as in E-MMSED-KK into (4) to get its diagonal element $f_{i, m}$ as

$$
f_{i, m}=\frac{\left[\frac{p_{1} K_{0}}{K_{1}}\right]^{\frac{1}{2}} h_{0, i, m}^{*} h_{i, m, K+1}^{*}}{\left[\sum_{k=1}^{K} \sum_{l=1}^{N}\left|h_{0, k, l}\right|^{2}\left|h_{k, l, K+1}\right|^{2}\left(p_{0}\left|h_{0, k, l}\right|^{2}+\sigma_{u}^{2}\right)\right]^{\frac{1}{2}}} .
$$

We note that in both (17) and (18), we have a double summation in the denominator instead of a single summation, when $K$ is greater than 1 . Also, we see that in both cases, the relay $\mathrm{R}_{i, m}$ needs forward CSI $h_{i, m, K+1}$.

\section{B. Selection of $K_{0}$}

Let us now select the best $K_{0}$ and use it while comparing its performance with MMSER- $\mu$ system. As it is hard to derive BER, we obtain SNR at D for any $K_{0}$ and attempt to select the value of $K_{0}$ that maximizes it.

In phase $k, k \in\left[K_{0}, K\right]$, D receives a scalar

$$
r_{K+1}^{(k)}=\mathbf{h}_{K+1} \mathbf{t}+u_{K+1}^{(k)},
$$

where $\mathbf{h}_{K+1}=\left[\mathbf{h}_{1, K+1}, \ldots, \mathbf{h}_{K, K+1}\right] \in \mathbb{C}^{1 \times K N}$. Substituting (16) into (19), we get

$$
\begin{aligned}
r_{K+1}^{(k)} & =\mathbf{h}_{K+1}\left[t_{0}^{\prime} \mathbf{F} \mathbf{h}_{0}+\frac{\mathbf{F}}{K_{0}} \sum_{i=0}^{K_{0}-1} \mathbf{u}^{(i)}\right]+u_{K+1}^{(k)} \\
& =r_{\text {sig }}^{(k)}+r_{\text {noi }}^{(k)}+u_{K+1}^{(k)},
\end{aligned}
$$

where

$r_{\mathrm{sig}}^{(k)}=\left[\frac{p_{0}}{K_{0}}\right]^{\frac{1}{2}} \mathbf{h}_{K+1} \mathbf{F} \mathbf{h}_{0} s$ and $r_{\text {noi }}^{(k)}=\frac{\mathbf{h}_{K+1} \mathbf{F}}{K_{0}} \sum_{i=0}^{K_{0}-1} \mathbf{u}^{(i)}$

are respectively, the signal and noise components of the received signal in phase $k$, without considering the noise that is added at $\mathrm{D}$. We do not take the noise added at D with these components, as it is not considered while deriving optimum precoder in MMSED. Now, we concatenate these components in D into vectors as $\mathbf{r}_{\mathrm{sig}}=\left[r_{\mathrm{sig}}^{\left(K_{0}\right)} \ldots r_{\mathrm{sig}}^{(K)}\right]^{T}$ and $\mathbf{r}_{\text {noi }}=\left[r_{\text {noi }}^{\left(K_{0}\right)} \ldots r_{\mathrm{noi}}^{(K)}\right]^{T}$.

The signal and noise powers from these vectors are defined as

$$
P_{S} \triangleq E\left[\mathbf{r}_{\mathrm{sig}}^{H} \mathbf{r}_{\mathrm{sig}}\right]=E\left[\sum_{k=K_{0}}^{K}\left|r_{\mathrm{sig}}^{(k)}\right|^{2}\right]
$$




$$
\text { and } P_{N} \triangleq E\left[\mathbf{r}_{\text {noi }}^{H} \mathbf{r}_{\text {noi }}\right]=E\left[\sum_{k=K_{0}}^{K}\left|r_{\text {noi }}^{(k)}\right|^{2}\right]
$$

respectively.

Claim 2: The ratio $P_{S} / P_{N}$ is given by

$$
\begin{aligned}
\frac{P_{S}}{P_{N}} & =\frac{p_{0} \sum_{i=1}^{K} \sum_{j=1}^{N}\left|h_{0, i, j}\right|^{4}}{\sigma_{u}^{2} \sum_{k=1}^{K} \sum_{l=1}^{N}\left|h_{0, k, l}\right|^{2}}, \\
\text { and } \frac{P_{S}}{P_{N}} & =\frac{p_{0} \sum_{i=1}^{K} \sum_{j=1}^{N}\left|h_{0, i, j}\right|^{4}\left|h_{i, j, K+1}\right|^{4}}{\sigma_{u}^{2} \sum_{k=1}^{K} \sum_{l=1}^{N}\left|h_{0, k, l}\right|^{2}\left|h_{k, l, K+1}\right|^{4}},
\end{aligned}
$$

for E-MMSED-KK and E-MMSED-L respectively.

Proof: See Appendix B.

From Claim 2, i.e., from (23) and (24), we see that we can select any $K_{0}$ from $[1, K]$ for both E-MMSED-KK and E-MMSED-L and these ratios do not vary. This also reflects in the BER plots shown in Section VI in Fig. 4 that for different values of $K_{0}$, BER does not change.

\section{Decoder at the Destination}

The signal received at the destination is given by

$$
\mathbf{r}_{D}=\left[\begin{array}{c}
r_{D_{1}} \\
\vdots \\
r_{D_{m}}
\end{array}\right]
$$

where $m$ is the total number of signals D has received in as many number of phases. For MMSER- $\mu, m=\mu, r_{D_{1}}=r_{K+1}^{(K+1-\mu)}$, and $r_{D_{m}}=r_{K+1}^{(K)}$. For E-MMSED schemes, $m=K_{1}=K-$ $K_{0}+1$ as seen in Section IV, $r_{D_{1}}=r_{K+1}^{\left(K_{0}\right)}$, and $r_{D_{m}}=r_{K+1}^{(K)}$. Let us write the decoded signal to be

$$
\hat{s} \triangleq \mathbf{f}_{D} \mathbf{r}_{D}
$$

where $\mathbf{f}_{D} \in \mathbb{C}^{1 \times m}$ is the decoder vector to be obtained by minimizing the MSE, $J_{D} \triangleq E|s-\hat{s}|^{2}$. The optimum $\mathbf{f}_{D}$ can be obtained as

$$
\hat{\mathbf{f}}_{D}=\mathbf{R}_{s^{\prime} r_{D}} \mathbf{R}_{r_{D}}^{-1}
$$

where

$$
\begin{aligned}
\mathbf{R}_{s^{\prime} r_{D}} & =E\left[\mathbf{r}_{D}^{H}\right] \in \mathbb{C}^{1 \times m} \\
\text { and } \mathbf{R}_{r_{D}} & =E\left[\begin{array}{lll}
R_{s^{\prime} r_{D_{1}}} & \cdots & R_{s^{\prime} r_{D_{m}}}
\end{array}\right] \\
& \left.\mathbf{r}_{D}^{H}\right] \in \mathbb{C}^{m \times m} \\
& {\left[\begin{array}{ccc}
R_{r_{D_{1}} r_{D_{1}}} & \cdots & R_{r_{D_{1}} r_{D_{m}}} \\
\vdots & \ddots & \vdots \\
R_{r_{D_{m}} r_{D_{1}}} & \cdots & R_{r_{D_{m}} r_{D_{m}}}
\end{array}\right] . }
\end{aligned}
$$

We note that, prime $\left(^{\prime}\right)$ is used over $s$ in correlation matrix $\mathbf{R}_{s^{\prime} r_{D}}$ to identify it to be a scalar unlike in (A2).

We use the decoder found in (27) in our simulations for both MMSER- $\mu$ and E-MMSED systems in Section VI.

\section{Simulation Results}

In this Section, we compare the BER performance of the proposed MMSER scheme with existing schemes and our enhanced E-MMSED schemes. We run Monte-Carlo simulations for both the cooperative and non-cooperative relays cases. First, we study the behavior of MMSER, E-MMSED-KK, and E-MMSED-L by varying the following parameters: total number of layers, $K$, number $K_{0}$ of transmissions by $\mathrm{S}$, and power $p_{0}$ allocated to $\mathrm{S}$. This is used to analyze the performance of MMSER and MMSED schemes and select the best values of $K_{0}$ and $p_{0}$ for E-MMSED in the simulations.

\section{A. Simulation Parameters}

We select $s$ from the Gray coded quadrature phase shift keying constellation with unit variance and use MMSE decoders at $\mathrm{D}$, derived in Section $\mathrm{V}$. In all the plots, we use $\mathrm{SNR}=10 \log \left(1 / \sigma_{u}^{2}\right)$ on the $x$-axis, where $\sigma_{u}^{2}$ is the variance of the noise added at the receivers.

For the simulations, we incorporate the signal-power path loss as in [26]; i.e., if $d_{i j}$ is the distance from $\mathrm{L}_{i}$ to $\mathrm{L}_{j}$, then the channel coefficient $h_{i, m, j, n}$ of the link $\ell_{i, m, j, n}$ is given by

$$
h_{i, m, j, n}=\frac{\xi_{i, m, j, n}}{d_{i j}^{\frac{\beta}{2}}},
$$

where $\left\{\xi_{i, m, j, n} \in \mathbb{C}: i, j \in[1, K], m, n \in[1, N]\right\}$ is an i.i.d. collection of random variables with $\xi_{i, m, j, n} \sim \mathcal{N}_{\mathbb{C}}(0,1)$ and $\beta$ is the path loss exponent. Hence, the variance of the channel coefficient $h_{i, m, j, n}$ is given by $1 / d_{i j}^{\beta}$.

Let us assume that the layers are equi-spaced, and hence the distance between any two layers $\mathrm{L}_{i}$ and $\mathrm{L}_{j}$ is $d_{i j}=(j-i) d /(K+1)$, where $d$ is the distance between $\mathrm{S}$ and D. Let $d=1$. Then, the channel variance of the link $h_{i, m, j, n}$ is $\operatorname{Var}\left[h_{i, m, j, n}\right]=1 / d_{i j}^{\beta}=(K+1)^{\beta} / \rho_{i j}^{\beta}$, where $\rho_{i j}=j-i$, and $\beta=2$. Though this special spatial structure is not required for operating our strategy, we use it for the simulations. More general scenarios can be considered, as we do not restrict the distance or the channel variance of the links in any manner, while deriving the optimum MMSER precoders. In all the simulations, whenever we need to increase the number of layers $K$, we do it by inserting them between $\mathrm{S}$ and $\mathrm{D}$ keeping the distance $d_{0, K+1}=d$ constant.

For $K=1$, Jing and Hassibi [1] proved that Jing-Hassibi Scheme (JHS) achieves maximum SNR at D when power is equally divided between $\mathrm{S}$ and the relays; i.e., $p_{0}=p_{1}=P / 2$. For $K=2$, we extended JHS (EJHS) [27] and proved that EJHS achieves maximum SNR at D when power is equally divided amongst $\mathrm{S}, \mathrm{L}_{1}$ and $\mathrm{L}_{2}$; i.e., $p_{0}=p_{1}=p_{2}=P / 3$. Extending this for any $K$, we use $p_{0}=P /(K+1)$ in all simulations for EJHS.

\section{B. Summary of Results}

- Figs. 2 and 3 show respectively how the performance of MMSER-1 worsens and that of MMSER-2 improves as $K$ increases. 


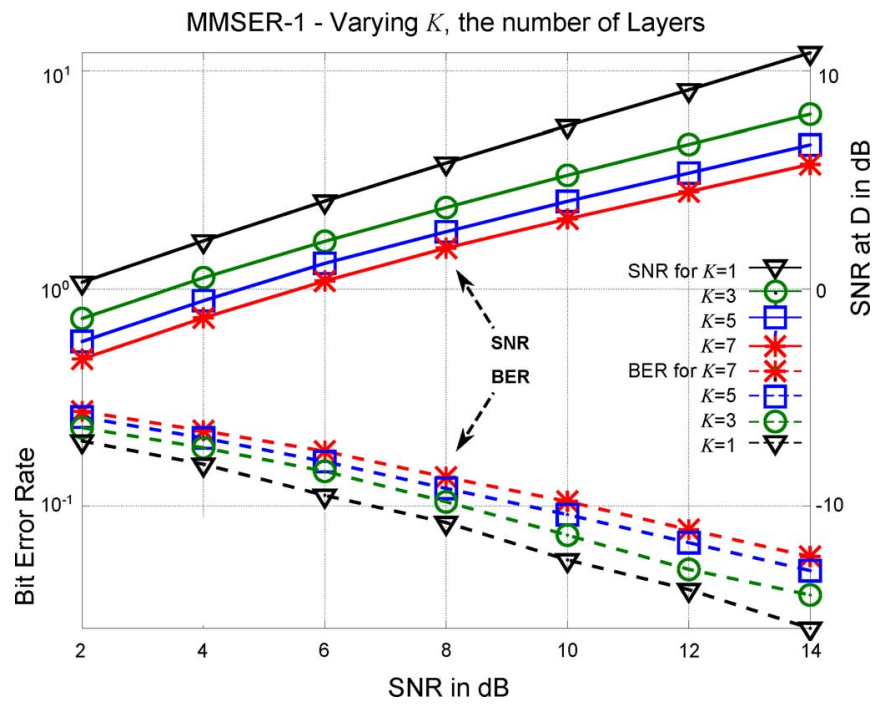

Fig. 2. Plots of SNR and BER of MMSER-1 for varying $K$, the number of layers with total power $P=1$ Watt.

- Figs. 4 and 5 show performance of E-MMSED-KK and L as a function of $K_{0}$ and $p_{0}$. This is used to select the best $K_{0}$ and $p_{0}$ for comparison with MMSER- $\mu$.

- Fig. 6 shows a comparison of BERs of MMSER-1 and MMSER-2 with MMSED for the single-layer case $(K=$ 1 ), when the relays cooperate.

— Figs. 7 and 8 show that the BER performance of MMSER outperforms that of E-MMSED-KK and approaches that of E-MMSED-L when $\mu$ is increased from 1 to $K+1$ with $K=3$ and 4 respectively, when the relays do not cooperate.

\section{Usefulness of Leaked Signals, $\mu>1$}

Fig. 2 shows SNR at D and BER plots of MMSER-1 when $K$ is varied. It can be observed that as $K$ increases, the SNR at $D$ decreases and BER of MMSER-1 increases. Fig. 3 shows BER plots of MMSER-2 for varying $K$. Unlike MMSER-1, the performance of MMSER-2 improves as the number of layers increases. This is because MMSER-2 uses leaked signals.

\section{Selection of $K_{0}$ and $p_{0}$ for E-MMSED-KK and $L$}

Fig. 4 shows the performance of E-MMSED-KK and E-MMSED-L, for various values of the number $K_{0}$ of transmissions of S. As was shown in (23) and (24), the BER plots also corroborate the fact that the performance does not vary with $K_{0}$. Hence, we use $K_{0}=1$ in all the simulations of E-MMSED.

Another parameter that needs to be fixed is the power allocated to $\mathrm{S} p_{0}$, and the relays $p_{r}=P-p_{0}$. We find these using simulations as shown in Fig. 5, where we have used $K=$ $4, N=2$, and $P=1$ Watt. For E-MMSED-KK, it can be seen that $20 \%$ of total power $P$ or $p_{0}=P /(K+1)=P / 5$ achieves low BER for SNR $\leq 10 \mathrm{~dB}$ and almost same BER for SNR $>10 \mathrm{~dB}$ than other power allocations. Similarly, $p_{0}=P / 2$ or $50 \%$ of total power achieves lowest BER for E-MMSED-L. Hence, we use these values for $p_{0}$ in all subsequent simulations for E-MMSED.

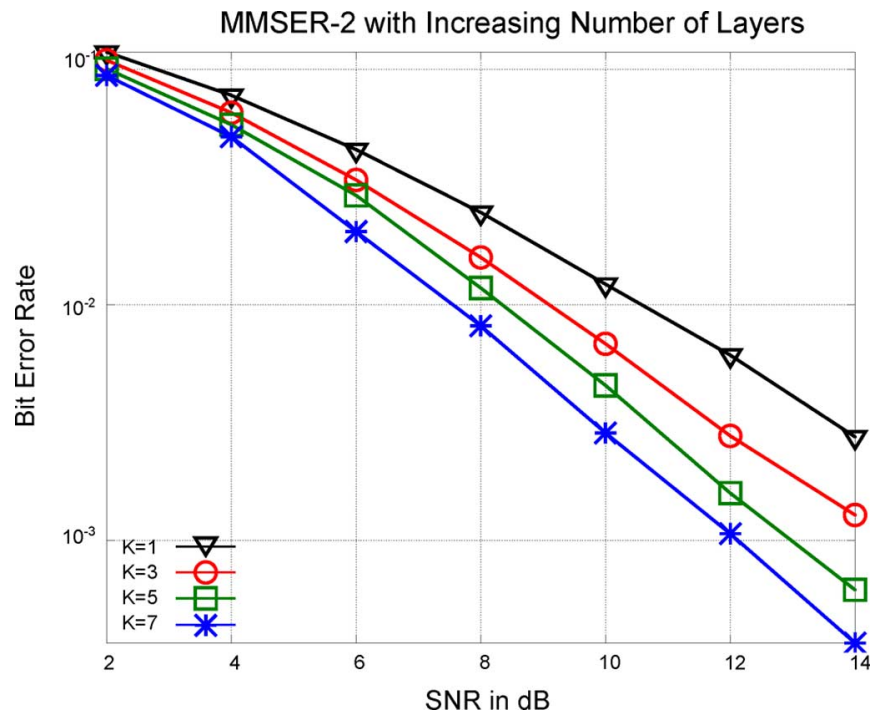

Fig. 3. BER plots of MMSER-2 for varying $K$, the number of layers with total power $P=1$ Watt. Unlike MMSER-1, BER performance improves as $K$ is increased due to the use of leaked signals.

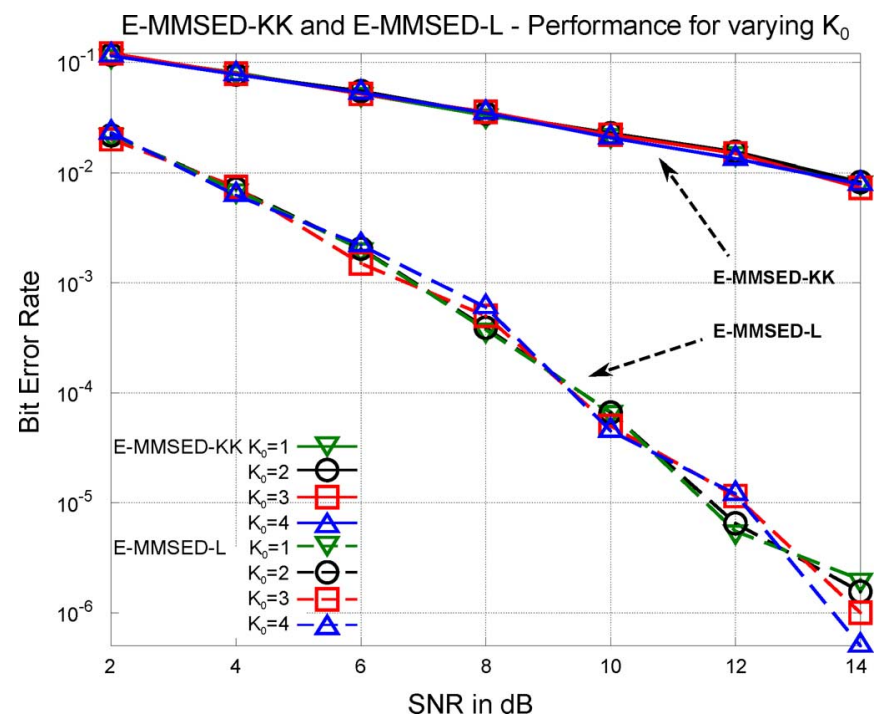

Fig. 4. E-MMSED-KK and E-MMSED-L showing same BER performance for varying $K_{0}$. Total power used in simulations is $P=1$ Watt.

\section{E. Comparison: Single Layer Case}

Fig. 6 shows plots of BER in a single layer network, when relays cooperate. We have used $P=2$ Watts for these simulations. We compare the performance of MMSER-1, MMSER-2, MMSED-K, and MMSED-K with leaked signals. For MMSED-K, we have generated the plot using Krishna equation (20) derived by Krishna et al. in [19]. We have also incorporated leaked signals in the MMSED-K scheme to obtain the MMSED-K with leak scheme.

MMSED schemes use global CSI optimally while MMSER- $\mu$ schemes use backward CSI alone. When there is only one relay $(N=1)$, MMSER-1 performs exactly same as that of MMSED. The performance of MMSER-2 and MMSED-K with leaked signals are also identical. This is a case where there is no advantage with forward CSI in the MMSED schemes. However, the use of leaked signals helps MMSER-2 


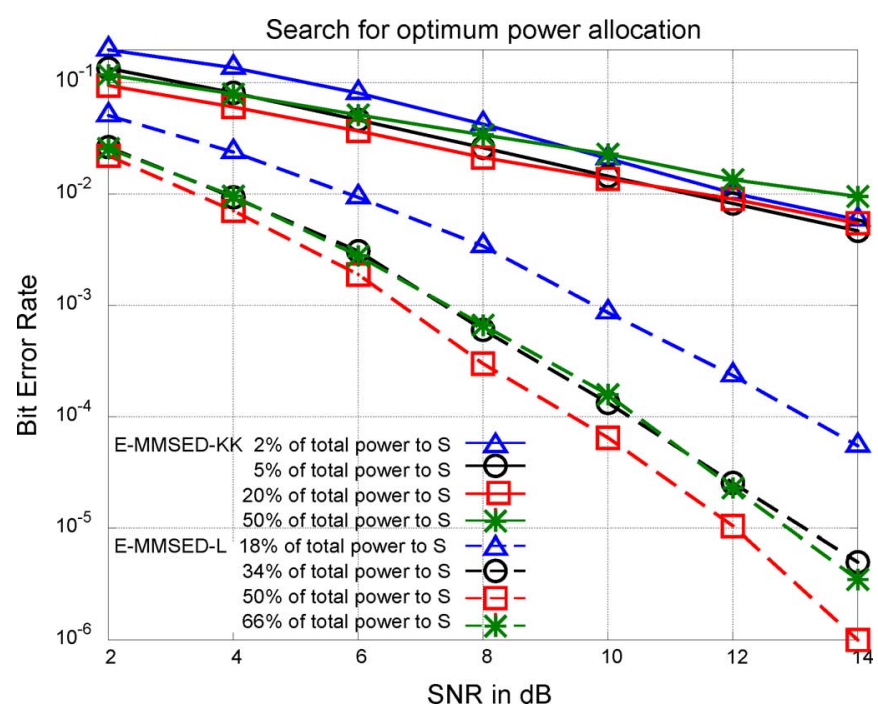

Fig. 5. Search for optimum power allocation for MMSED. Shows that when power is equally distributed to $\mathrm{S}$ and layers, BER performance of E-MMSED-KK is the best and when $50 \%$ of power is allocated to $\mathrm{S}$, E-MMSED-L attains best BER performance. Total power used in simulations is $P=1$ Watt.

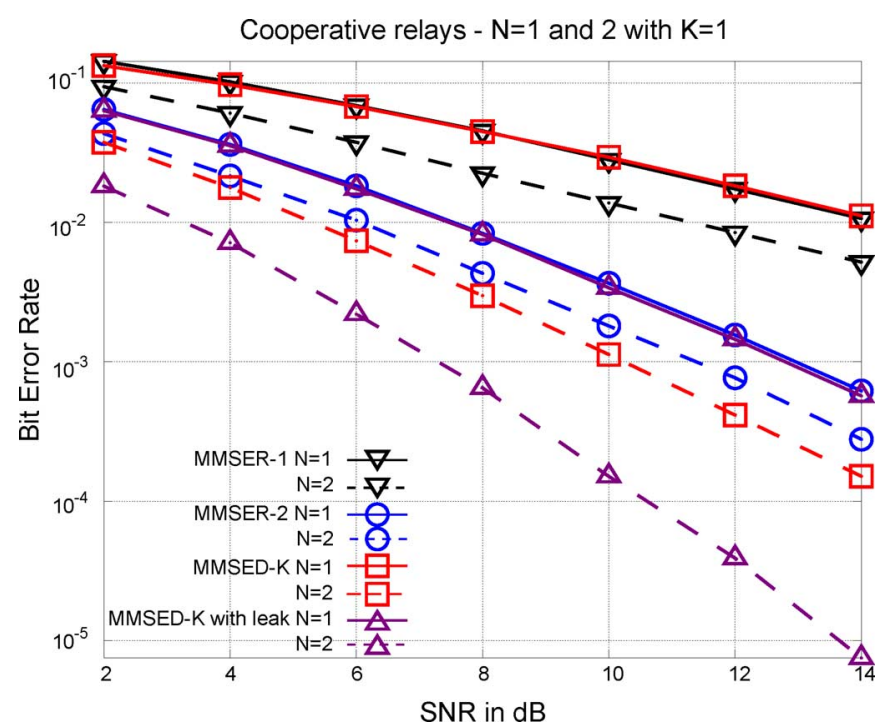

Fig. 6. Cooperative relays performance comparison. Performances of MMSER-1 and MMSED-K are the same when $N=1$. Similarly, the performance of MMSED-K 'with leak' is the same as that of MMSER-2. For $N=2$, the performance of MMSED-K is better than MMSER-1 and MMSER-2 schemes. Total power used in simulations is $P=2$ Watts.

and MMSED-K with leaked signals perform better than the MMSER-1 and MMSED-K schemes.

When there are 2 relays, the MMSED scheme performance improves significantly because forward CSI can be used to achieve beamforming gain in the transmission from the relay layer to the destination. In this case, MMSER-2 is able to bridge a significant part of the gap between MMSER-1 and MMSED using the leaked signal from $\mathrm{S}$ at $\mathrm{D}$. The MMSED-K scheme with leaked signals further improves upon the MMSED-K scheme.

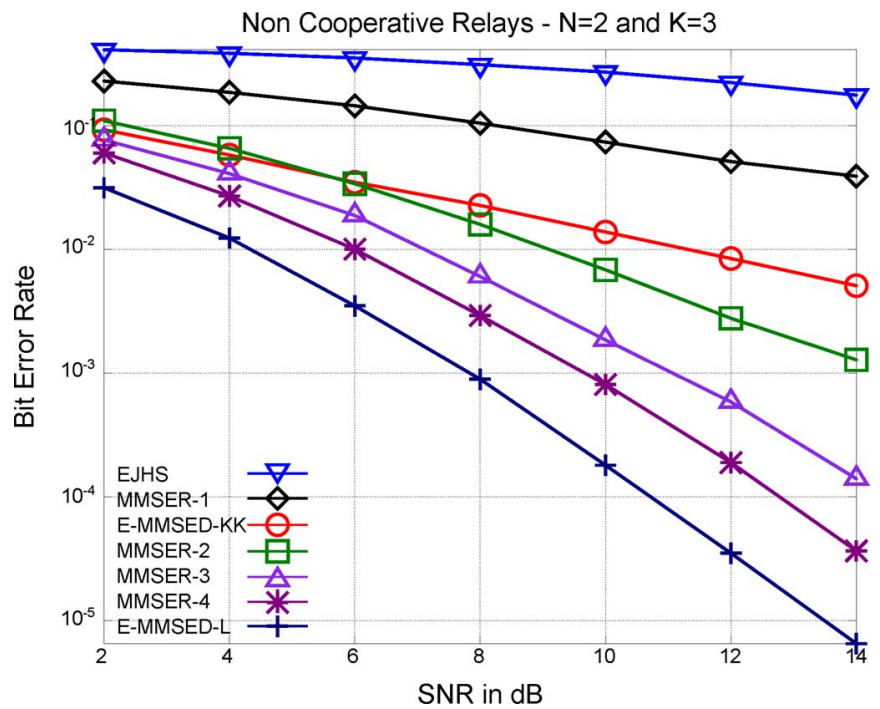

Fig. 7. BER plots of EJHS, E-MMSED, and MMSER- $\mu, \mu \in[1-4]$. Performance of MMSER- $\mu$ is better than E-MMSED-KK when $\mu \geq 2$ and it approaches that of E-MMSED-L when $\mu$ is increased. Total power used in simulations is $P=1$ Watt.

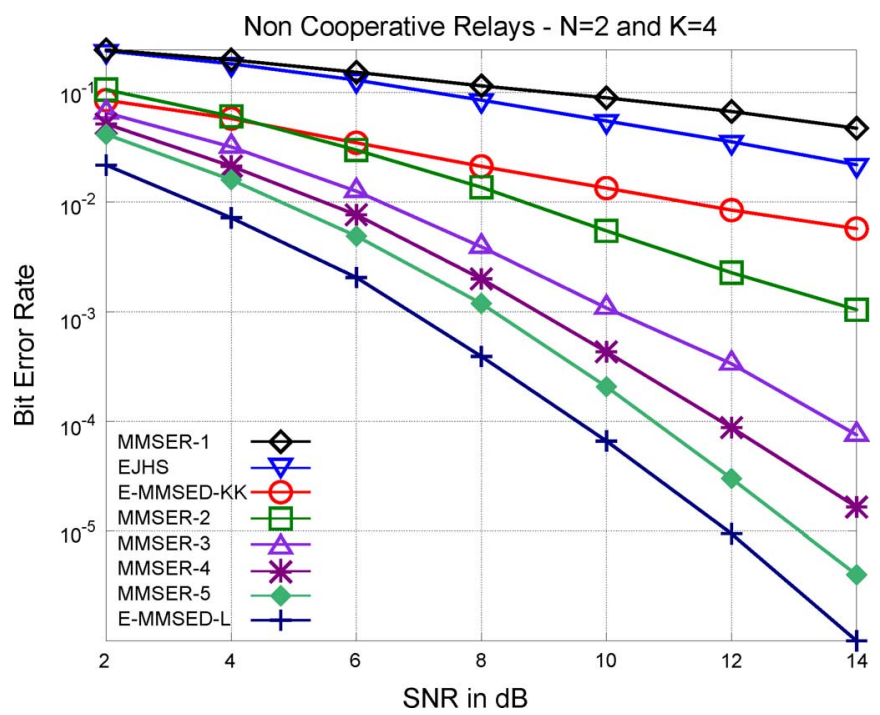

Fig. 8. BER plots of EJHS, E-MMSED, and MMSER- $\mu, \mu \in[1-5]$. Performance of MMSER- $\mu$ is better than E-MMSED-KK when $\mu \geq 2$ and it approaches that of E-MMSED-L when $\mu$ is increased. Total power used in simulations is $P=1$ Watt.

\section{F. Comparison: Multi-Layer Case}

Finally, we consider 3 and 4 layer systems with number of relays in each layer to be $N=2$ and a total transmitted power of $P=1$ Watt for comparing BER performance of the proposed system MMSER, with E-MMSED systems. Ideally, we would like to compare the MMSER scheme with the MMSED scheme. However, the MMSED solution is known only for the one layer case (in closed-form) and two layer case (as an iterative solution). Therefore, we cannot compare with MMSED when the number of layers $(K)$ is 3 or 4. In Figs. 7 and 8, we show the BER plots of EJHS, MMSER- $\mu, \mu=1$ to 5 , E-MMSED-KK and E-MMSED-L when $K=3$ and 4 respectively.

In both Figs. 7 and 8, we observe that there is an advantage of $6 \mathrm{~dB}$ of SNR at BER $=10^{-1}$ for MMSER-2 over 
MMSER-1. MMSER-3 has an advantage of $2 \mathrm{~dB}$ of SNR at $\mathrm{BER}=10^{-2}$ than MMSER-2. Among the two E-MMSED schemes developed, the E-MMSED-L scheme uses global CSI more effectively. Our MMSER- $\mu$ scheme performs better than E-MMSED-KK scheme even though we do not use forward CSI, and worse compared to the E-MMSED-L scheme. MMSER- $\mu$ approaches the performance of E-MMSED-L using leaked signals. For example, at BER $=10^{-3}$, the advantage of E-MMSED-L over MMSER comes down from 2 to $1 \mathrm{~dB}$ of SNR, when $\mu$ is increased from 4 to 5 . The lack of forward CSI in MMSER- $\mu$ is compensated using leaked signals at the relays.

The comparisons for the multi-layer case in Figs. 7 and 8 also show much more gain (than in the single-layer case) using global CSI in terms of the gap between MMSER-1 and the E-MMSED-L schemes. Therefore, the gain from global CSI seems to increase when the number of layers increases. However, using more leaked signals reduces this gap significantly. In Figs. 7 and 8, MMSER-4 and MMSER-5 seem to approach the E-MMSED-L scheme respectively.

\section{SUMmARY AND CONCLUSION}

In this paper, we have considered the AF relaying protocol for a multi-layer cooperative system, and proposed a precoder design method MMSER which minimizes the MSE at each relay instead of the MSE at the destination that is considered in earlier works. Whereas MMSED is a difficult optimization problem using global CSI and even an iterative numerical solution is not available for more than two layers (to the best of our knowledge), our approach needs only backward CSI and results in layer-wise optimization that yields closed form solution for any number of relay layers even when leaked signals are considered. Since MMSED precoder solutions are not available for more than two layers, for comparison, we have proposed E-MMSED schemes, that are suboptimal, for multiple layers. These schemes may be of independent interest. Though MMSER uses a suboptimal cost function, its performance is shown to exceed/approach the performance of the proposed E-MMSED schemes. We believe that our MMSER schemes provide an interesting method for $\mathrm{AF}$ precoder design for multi-layer relay system.

We found the evaluation of the achieved MSE at the destination for our MMSER schemes challenging, and thus resorted to simulation based study. Analytical performance evaluation remains a valuable future work.

\section{APPENDIX}

Proof of Claim 1: Optimum Precoder at $\mathrm{L}_{k}$

Expanding (5), we get

$$
\begin{aligned}
J_{k}\left(\mathbf{F}_{k}\right) & =E\left[\mathbf{s}^{H} \mathbf{s}-2 \Re\left(\mathbf{r}_{k}^{H} \mathbf{F}_{k}^{H} \mathbf{s}\right)+\mathbf{r}_{k}^{H} \mathbf{F}_{k}^{H} \mathbf{F}_{k} \mathbf{r}_{k}\right] \\
& =N-2 \Re\left(\operatorname{Tr}\left[\mathbf{F}_{k}^{H} \mathbf{R}_{s r_{k}}\right]\right)+\operatorname{Tr}\left[\mathbf{F}_{k}^{H} \mathbf{F}_{k} \mathbf{R}_{r_{k}}\right],
\end{aligned}
$$

where $\Re(\cdot)$ is the real-part and $\operatorname{Tr}[\cdot]$ denotes the trace operator. To arrive at (A1), we have used $\mathbf{r}_{k}^{H} \mathbf{F}_{k}^{H} \mathbf{F}_{k} \mathbf{r}_{k}=$ $\operatorname{Tr}\left(\mathbf{r}_{k}^{H} \mathbf{F}_{k}^{H} \mathbf{F}_{k} \mathbf{r}_{k}\right)$ as it is a scalar and the cyclic properties of the $\operatorname{Tr}(\cdot)$ function namely, $\operatorname{Tr}(\mathbf{A B C})=\operatorname{Tr}(\mathbf{B C A})$. Here, the correlation matrices $\mathbf{R}_{s r_{k}}$ and $\mathbf{R}_{r_{k}}$ are given by

$$
\begin{aligned}
\mathbf{R}_{s r_{k}} & =E\left[\mathbf{s r}_{k}^{H}\right]=E\left[\mathbf{s}\left(\mathbf{r}_{k}^{(n) H}, \ldots, \mathbf{r}_{k}^{(k-1) H}\right)\right] \\
& =\left[\mathbf{R}_{s r_{k}^{(n)}}, \ldots, \mathbf{R}_{s r_{k}^{(k-1)}}\right]
\end{aligned}
$$

and

$$
\begin{aligned}
\mathbf{R}_{r_{k}} & =E\left[\mathbf{r}_{k} \mathbf{r}_{k}^{H}\right] \\
& =\left[\begin{array}{ccc}
\mathbf{R}_{r_{k}^{(n)} r_{k}^{(n)}} & \cdots & \mathbf{R}_{r_{k}^{(n)} r_{k}^{(k-1)}} \\
\vdots & \ddots & \vdots \\
\mathbf{R}_{r_{k}^{(k-1)} r_{k}^{(n)}} & \cdots & \mathbf{R}_{r_{k}^{(k-1)} r_{k}^{(k-1)}}
\end{array}\right]
\end{aligned}
$$

respectively, where $n=[k-\mu]^{+}$. Also, $\mathbf{R}_{s r_{k}^{(i)}}$ and $\mathbf{R}_{r_{k}^{(i)} r_{k}^{(j)}}, i, j \in[n, k-1]$, are given by

$$
\mathbf{R}_{s r_{k}^{(i)}}=E\left[\mathbf{s r}_{k}^{(i) H}\right] \text { and } \mathbf{R}_{r_{k}^{(i)} r_{k}^{(j)}}=E\left[\mathbf{r}_{k}^{(i)} \mathbf{r}_{k}^{(j) H}\right]
$$

respectively, which can be found using Table I. Now, expanding (6), we get

$$
C_{k}\left(\mathbf{F}_{k}\right)=\operatorname{Tr}\left(\mathbf{F}_{k}^{H} \mathbf{F}_{k} \mathbf{R}_{r_{k}}\right)-p_{k} .
$$

Substituting (A1) and (A4) into (7), we get

$$
\begin{aligned}
\mathcal{L}_{k}\left(\mathbf{F}_{\mathbf{k}}, \lambda_{k}\right)=N-2 \Re( & \left.\operatorname{Tr}\left[\mathbf{F}_{k}^{H} \mathbf{R}_{s r_{k}}\right]\right)+\operatorname{Tr}\left(\mathbf{F}_{k}^{H} \mathbf{F}_{k} \mathbf{R}_{r_{k}}\right) \\
& +\lambda_{k}\left[\operatorname{Tr}\left(\mathbf{F}_{k}^{H} \mathbf{F}_{k} \mathbf{R}_{r_{k}}\right)-p_{k}\right] .
\end{aligned}
$$

Now, we will derive $\hat{\mathbf{F}}_{k}$ for the case when the relays are cooperative.

1) Cooperative Relays: Here, each of the sub-matrices $\mathbf{F}_{k, i} \in \mathbb{C}^{N \times N}$ shown in (1) are nondiagonal as mentioned earlier. Differentiating (A5) w.r.t. $\mathbf{F}_{k}^{*}$ [28] and using complementary slackness [24] yield

$$
\nabla_{\mathbf{F}_{k}^{*}} \mathcal{L}_{k}=-\mathbf{R}_{s r_{k}}+\mathbf{F}_{k} \mathbf{R}_{r_{k}}+\lambda_{k} \mathbf{F}_{k} \mathbf{R}_{r_{k}}
$$

and

$$
\lambda_{k}\left[\operatorname{Tr}\left(\mathbf{F}_{k}^{H} \mathbf{F}_{k} \mathbf{R}_{r_{k}}\right)-p_{k}\right]=0,
$$

respectively. Equating (A6) to zero, we get

$$
\mathbf{F}_{k}=\frac{1}{1+\lambda_{k}} \mathbf{R}_{s r_{k}} \mathbf{R}_{r_{k}}^{-1} .
$$

From (A7), we have either $\lambda_{k}=0$ or

$$
p_{k}=\operatorname{Tr}\left(\mathbf{F}_{k}^{H} \mathbf{F}_{k} \mathbf{R}_{r_{k}}\right) .
$$

If the unconstrained estimate $\mathbf{F}_{k}=\mathbf{R}_{s r_{k}} \mathbf{R}_{r_{k}}^{-1}$ satisfies the power constraint, then it is also the solution to the MMSE problem with the power constraint, and $\lambda_{k}=0$. Otherwise, $\lambda_{k}>0$, and substituting (A8) into (A9) and rearranging, we get

$$
\left(1+\lambda_{k}\right)^{2}=\frac{1}{p_{k}} \operatorname{Tr}\left[\mathbf{R}_{s r_{k}}^{H} \mathbf{R}_{s r_{k}} \mathbf{R}_{r_{k}}^{-1}\right]
$$

as $\left(\mathbf{R}_{r_{k}}^{-1}\right)^{H}=\mathbf{R}_{r_{k}}^{-1}$ and $\operatorname{Tr}(\mathbf{A B C})=\operatorname{Tr}(\mathbf{B C A})$. Substituting (A10) into (A8), we get the optimum precoder as shown in (8). This is simply the scaled version of the unconstrained 
MMSE estimate scaled such that the power constraint is met with equality.

Even when $\lambda_{k}=0$, i.e., when the unconstrained estimate has lower power, we amplify the estimate to use the full sum transmit power for each layer. This is because it is optimal to transmit using the full power for optimal estimation performance at the next layer. Therefore, we always use the precoder specified by (8).

2) Non-Cooperative Relays: Here each of the sub-matrices $\mathbf{F}_{k, i} \in \mathbb{C}^{N \times N}$ shown in (1) are constrained to be diagonal. To simplify (A5), let us consider

$$
\begin{aligned}
\mathbf{F}_{k}^{H} \mathbf{F}_{k} \mathbf{R}_{r_{k}} & \\
= & {\left[\begin{array}{c}
\mathbf{F}_{k, n}^{H} \\
\vdots \\
\mathbf{F}_{k, k-1}^{H}
\end{array}\right]\left[\mathbf{F}_{k, n}, \ldots, \mathbf{F}_{k, k-1}\right] \mathbf{R}_{r_{k}} } \\
= & {\left[\begin{array}{ccc}
\mathbf{F}_{k, n}^{H} \mathbf{F}_{k, n} & \cdots & \mathbf{F}_{k, n}^{H} \mathbf{F}_{k, k-1} \\
\vdots & \ddots & \vdots \\
\mathbf{F}_{k, k-1}^{H} \mathbf{F}_{k, n} & \cdots & \mathbf{F}_{k, k-1}^{H} \mathbf{F}_{k, k-1}
\end{array}\right] \mathbf{R}_{r_{k}} }
\end{aligned}
$$

Substituting $\mathbf{R}_{r_{k}}$ from (A3) into (A11) we get

$$
\begin{aligned}
\mathbf{F}_{k}^{H} \mathbf{F}_{k} \mathbf{R}_{r_{k}} & =\left[\begin{array}{ccc}
\boldsymbol{\Gamma}_{k, n, n} & \cdots & \boldsymbol{\Gamma}_{k, n, k-1} \\
\vdots & \ddots & \vdots \\
\boldsymbol{\Gamma}_{k, k-1, n} & \cdots & \boldsymbol{\Gamma}_{k, k-1, k-1}
\end{array}\right], \\
\text { where } \boldsymbol{\Gamma}_{k, i^{\prime}, j^{\prime}} & =\sum_{i=n}^{k-1} \mathbf{F}_{k, i^{\prime}}^{H} \mathbf{F}_{k, i} \mathbf{R}_{r_{k}^{(i)} r_{k}^{\left(j^{\prime}\right)}} .
\end{aligned}
$$

Taking the trace of (A12), we get

$$
\begin{aligned}
\operatorname{Tr}\left(\mathbf{F}_{k}^{H} \mathbf{F}_{k} \mathbf{R}_{r_{k}}\right) & =\sum_{j=n}^{k-1} \operatorname{Tr}\left(\boldsymbol{\Gamma}_{k, j, j}\right) \\
& =\sum_{j=n}^{k-1} \sum_{i=n}^{k-1} \operatorname{Tr}\left(\mathbf{F}_{k, j}^{H} \mathbf{F}_{k, i} \mathbf{R}_{r_{k}^{(i)} r_{k}^{(j)}}\right) \\
& =\sum_{j=n}^{k-1} \sum_{i=n}^{k-1} \sum_{l=1}^{N} f_{k, j, l}^{*} f_{k, i, l} \gamma_{k l}^{(i)(j)}
\end{aligned}
$$

where $f_{k, i, l}, f_{k, j, l}$, and $\gamma_{k l}^{(i)(j)}$ represent the $l$ th diagonal elements of $\mathbf{F}_{k, i}, \mathbf{F}_{k, j}$ and $\mathbf{R}_{r_{k}^{(i)} r_{k}^{(j)}}$ respectively. To arrive at (A13), we used the fact that the matrices $\mathbf{F}_{k, i}, i \in[n, k-1]$ are diagonal. Finally, to simplify (A5) we find $\mathbf{F}_{k}^{H} \mathbf{R}_{s r_{k}}$ as

$$
\begin{aligned}
\mathbf{F}_{k}^{H} \mathbf{R}_{s r_{k}}= & {\left[\begin{array}{c}
\mathbf{F}_{k, n}^{H} \\
\vdots \\
\mathbf{F}_{k, k-1}^{H}
\end{array}\right]\left[\begin{array}{lll}
\mathbf{R}_{s r_{k}^{(n)}} & \cdots & \mathbf{R}_{s r_{k}^{(k-1)}}
\end{array}\right] } \\
= & {\left[\begin{array}{ccc}
\mathbf{F}_{k, n}^{H} \mathbf{R}_{s r_{k}^{(n)}} & \cdots & \mathbf{F}_{k, n}^{H} \mathbf{R}_{s r_{k}^{(k-1)}} \\
\vdots & \ddots & \vdots \\
\mathbf{F}_{k, k-1}^{H} \mathbf{R}_{s r_{k}^{(n)}} & \cdots & \mathbf{F}_{k, k-1}^{H} \mathbf{R}_{s r_{k}^{(k-1)}}
\end{array}\right] . }
\end{aligned}
$$

Taking the trace of (A14), we get

$$
\operatorname{Tr}\left(\mathbf{F}_{k}^{H} \mathbf{R}_{s r_{k}}\right)=\sum_{j=n}^{k-1} \operatorname{Tr}\left(\mathbf{F}_{k, j}^{H} \mathbf{R}_{s r_{k}^{(j)}}\right)=\sum_{j=n}^{k-1} \sum_{l=1}^{N} f_{k, j, l}^{*} \gamma_{k l}^{s(j)},
$$

where $\gamma_{k l}^{s(j)}$ is the $l$ th diagonal element of the correlation matrix $\mathbf{R}_{s r_{k}^{(j)}}$. From (A13) and (A15), (A5) becomes

$$
\begin{aligned}
\mathcal{L}_{k}= & N-2 \Re\left[\sum_{j=n}^{k-1} \sum_{l=1}^{N} f_{k, j, l}^{*} \gamma_{k l}^{s(j)}\right] \\
& +\left(1+\lambda_{k}\right) \sum_{j=n}^{k-1} \sum_{i=n}^{k-1} \sum_{l=1}^{N} f_{k, j, l}^{*} f_{k, i, l} \gamma_{k l}^{(i)(j)}-\lambda_{k} p_{k}
\end{aligned}
$$

Differentiating (A16) w.r.t. the conjugate of the precoder matrix diagonal element, $f_{k, j, l}^{*}, j \in[n, k-1], l \in[1, N]$ and simplifying, we get

$$
\sum_{i=n}^{k-1} f_{k, i, l} \gamma_{k l}^{(i)(j)}=\frac{\gamma_{k l}^{s(j)}}{1+\lambda_{k}}
$$

From complementary slackness, we get $\lambda_{k}=0$ or

$$
\begin{aligned}
p_{k} & =\sum_{j=n}^{k-1} \sum_{i=n}^{k-1} \sum_{l=1}^{N} f_{k, j, l}^{*} f_{k, i, l} \gamma_{k l}^{(i)(j)} \\
& =\sum_{l=1}^{N} \operatorname{Tr}\left(\mathbf{f}_{k, l}^{H} \mathbf{f}_{k, l} \gamma_{k, l}\right) .
\end{aligned}
$$

Equation (A17) can be written in matrix form as

$$
\mathbf{f}_{k, l} \Upsilon_{k l}=\frac{1}{1+\lambda_{k}} \Upsilon_{k l}^{s}, l \in[1, N]
$$

where $\mathbf{f}_{k, l}, \Upsilon_{k l}^{s}$, and $\Upsilon_{k l}$ are as defined in (10), (11), and (12) respectively. From (A19), we get

$$
\mathbf{f}_{k, l}=\frac{1}{1+\lambda_{k}} \Upsilon_{k l}^{s} \Upsilon_{k l}-1
$$

as $\Upsilon_{k l}$ is a nonsingular matrix which depends on $\mathbf{R}_{s r_{k}}$ and $\mathbf{R}_{r_{k}}$. Proceeding (as in the cooperative relays case) with (A18) and (A20), we get the required (9).

\section{APPENDIX}

\section{Proof of Claim 2: SNR of E-MMSED}

Using (20), $P_{S}$ in (21) can be expanded as

$P_{S}=\frac{p_{0} K_{1}}{K_{0}} \mathbf{h}_{0}^{H} \mathbf{F}^{H} \mathbf{h}_{K+1}^{H} \mathbf{h}_{K+1} \mathbf{F} \mathbf{h}_{0}$ where $K_{1}=K-K_{0}+1$

$$
=\frac{p_{0} K_{1}}{K_{0}} \sum_{i=1}^{K} \sum_{j=1}^{N}\left|h_{0, i, j}\right|^{2}\left|h_{i, j, K+1}\right|^{2}\left|f_{i, j}\right|^{2}
$$

using the diagonal properties of $\mathbf{F}$ and $E\left(|s|^{2}\right)=1$. Similarly, from (20) we can write

$$
\begin{aligned}
E\left|r_{\text {noi }}^{(k)}\right|^{2} & =E\left[\frac{\mathbf{h}_{K+1} \mathbf{F}}{K_{0}} \sum_{i=0}^{K_{0}-1} \mathbf{u}^{(i)}\right]^{H}\left[\frac{\mathbf{h}_{K+1} \mathbf{F}}{K_{0}} \sum_{j=0}^{K_{0}-1} \mathbf{u}^{(j)}\right] \\
& =\frac{\sigma_{u}^{2}}{K_{0}} \mathbf{h}_{K+1} \mathbf{F} \mathbf{F}^{H} \mathbf{h}_{K+1}^{H} \\
& =\frac{\sigma_{u}^{2}}{K_{0}} \sum_{i=1}^{K} \sum_{j=1}^{N}\left|h_{i, j, K+1}\right|^{2}\left|f_{i, j}\right|^{2} .
\end{aligned}
$$


Hence from (22), $P_{N}$ can be written as

$$
P_{N}=\frac{\sigma_{u}^{2} K_{1}}{K_{0}} \sum_{i=1}^{K} \sum_{j=1}^{N}\left|h_{i, j, K+1}\right|^{2}\left|f_{i, j}\right|^{2}
$$

\section{A. E-MMSED-KK}

Substituting (17) into (A21), we get

$$
P_{S}=\frac{p_{0} p_{1} \sum_{i=1}^{K} \sum_{j=1}^{N}\left|h_{0, i, j}\right|^{4}}{\sum_{k=1}^{K} \sum_{l=1}^{N} \frac{\left|h_{0, k, l}\right|^{2}}{\left|h_{k, l, K+1}\right|^{2}}\left(p_{0}\left|h_{0, k, l}\right|^{2}+\sigma_{u}^{2}\right)} .
$$

Similarly, substituting (17) into (A22), we get

$$
P_{N}=\frac{\sigma_{u}^{2} p_{1} \sum_{i=1}^{K} \sum_{j=1}^{N}\left|h_{0, i, j}\right|^{2}}{\sum_{k=1}^{K} \sum_{l=1}^{N} \frac{\left|h_{0, k, l}\right|^{2}}{\left|h_{k, l, K+1}\right|^{2}}\left(p_{0}\left|h_{0, k, l}\right|^{2}+\sigma_{u}^{2}\right)} .
$$

Dividing (A23) by (A24), we get (23).

\section{B. E-MMSED-L}

Substituting (18) into (A21), we get

$$
P_{S}=\frac{p_{0} p_{1} \sum_{i=1}^{K} \sum_{j=1}^{N}\left|h_{0, i, j}\right|^{4}\left|h_{i, j, K+1}\right|^{4}}{\sum_{k=1}^{K} \sum_{l=1}^{N}\left|h_{0, k, l}\right|^{2}\left|h_{k, l, K+1}\right|^{2}\left(p_{0}\left|h_{0, k, l}\right|^{2}+\sigma_{u}^{2}\right)} .
$$

Similarly, substituting (18) into (A22), we get

$$
P_{N}=\frac{\sigma_{u}^{2} p_{1} \sum_{i=1}^{K} \sum_{j=1}^{N}\left|h_{0, i, j}\right|^{2}\left|h_{i, j, K+1}\right|^{4}}{\sum_{k=1}^{K} \sum_{l=1}^{N}\left|h_{0, k, l}\right|^{2}\left|h_{k, l, K+1}\right|^{2}\left(p_{0}\left|h_{0, k, l}\right|^{2}+\sigma_{u}^{2}\right)} .
$$

Dividing (A25) by (A26), we get (24).

\section{REFERENCES}

[1] Y. Jing and B. Hassibi, "Distributed space-time coding in wireless relay networks," IEEE Trans. Wireless Commun., vol. 5, pp. 3524-3536, Dec. 2006.

[2] Y. Jing and B. Hassibi, "Cooperative diversity in wireless relay networks with multiple-antenna nodes," in Proc. Int. Symp. Inf. Theory (ISIT), Sep. 2005, pp. 815-819.

[3] Y. Jing and B. Hassibi, "Diversity analysis of distributed space-time codes in relay networks with multiple transmit/receive antennas," EURASIP J. Adv. Signal Process., vol. 2008, pp. 1-17, Jan. 2008.

[4] A. Sendonaris, E. Erkip, and B. Aazhang, "User cooperation diversity. Part I. System description," IEEE Trans. Commun., vol. 51, pp. 1927-1938, Nov. 2003.

[5] A. Sendonaris, E. Erkip, and B. Aazhang, "User cooperation diversity. Part II. Implementation aspects and performance analysis," IEEE Trans. Commun., vol. 51, pp. 1939-1948, Nov. 2003.

[6] G. J. Pottie and W. J. Kaiser, "Wireless integrated network sensors," Commun. ACM, vol. 43, pp. 51-58, May 2000.

[7] S. Borade, L. Zheng, and R. Gallager, "Amplify-and-forward in wireless relay networks: Rate, diversity, and network size," IEEE Trans. Inf. Theory, vol. 53, pp. 3302-3318, Oct. 2007.

[8] J. Laneman and G. Wornell, "Distributed space-time-coded protocols for exploiting cooperative diversity in wireless networks," IEEE Trans. Inf. Theory, vol. 49, pp. 2415-2425, Oct. 2003.

[9] T. E. Hunter and A. Nosratinia, "Diversity through coded cooperation," IEEE Trans. Wireless Commun., vol. 5, pp. 283-289, Feb. 2006.

[10] T. Cover and A. E. Gamal, "Capacity theorems for the relay channel," IEEE Trans. Inf. Theory, vol. 25, pp. 572-584, Sep. 1979.
[11] G. Kramer, M. Gastpar, and P. Gupta, "Cooperative strategies and capacity theorems for relay networks," IEEE Trans. Inf. Theory, vol. 51, pp. 3037-3063, Sep. 2005.

[12] A. Gamal and M. Aref, "The capacity of the semi-deterministic relay channel," IEEE Trans. Inf. Theory, vol. 28, p. 536, May 1982.

[13] E. Chiu, V. K. N. Lau, S. Zhang, and B. S. M. Mok, "Precoder design for multi-antenna partial decode-and-forward (PDF) cooperative systems with statistical CSIT and MMSE-SIC receivers," IEEE Trans. Wireless Commun., vol. 11, pp. 1343-1349, Apr. 2012.

[14] V. Tarokh, H. Jafarkhani, and A. R. Calderbank, "Space-time block codes from orthogonal designs," IEEE Trans. Inf. Theory, vol. 45, pp. 1456-1467, Jul. 1999.

[15] Y. Ding, J.-K. Zhang, and K. M. Wong, "The amplify-and-forward half-duplex cooperative system: Pairwise error probability and precoder design," IEEE Trans. Signal Process., vol. 55, pp. 605-617, Feb. 2007.

[16] Y. Ding, J.-K. Zhang, and K. M. Wong, "Optimal precoder for amplify-and-forward half-duplex relay system," IEEE Trans. Wireless Commun., vol. 7, pp. 2890-2895, Aug. 2008.

[17] K. Gomadam and S. Jafar, "The effect of noise correlation in amplify-and-forward relay networks," IEEE Trans. Inf. Theory, vol. 55, pp. 731-745, Feb. 2009.

[18] N. Khajehnouri and A. H. Sayed, "Distributed MMSE relay strategies for wireless sensor networks," IEEE Trans. Signal Process., vol. 55, pp. 3336-3348, Jul. 2007.

[19] R. Krishna, Z. Xiong, and S. Lambotharan, "A cooperative MMSE relay strategy for wireless sensor networks," IEEE Signal Process. Lett., vol. 15, pp. 549-552, Jul. 2008.

[20] K. Lee, H. M. Kwon, Y. Ding, Z. Wang, Y. Bi, and Y. Ibdah, "Amplifying matrix design for cooperative relay networks under channel uncertainty and power constraint," in Proc. 7th Int. Conf. Wireless Mobile Commun. (ICWMC), Jun. 2011, pp. 139-144.

[21] K. Lee, H. M. Kwon, and Z. Wang, "Noncooperative distributed wireless relay networks under channel uncertainty and power constraint," in Proc. 20th Int. Conf. Comput. Commun. Netw. (ICCCN), Aug. 2011, pp. 1-5.

[22] K. Lee, H. M. Kwon, H. Kim, E. M. Sawan, H. Park, and Y. H. Lee, "AF MIMO wireless relay networks under received power constraint," in Proc. 75th Veh. Technol. Conf. (VTC Fall), Sep. 2012, pp. 1-5.

[23] K. Lee, H. M. Kwon, W. Xiong, H. Kim, S. Feng, H. Park, and Y. H. Lee, "Two-level MMSE relay strategy for an AF wireless relay network," in Proc. 50th Ann. Allerton Conf. Commun., Contr., Comput., Oct. 2012, pp. 1653-1658.

[24] S. Boyd and L. Vandenberghe, Convex Optimization. Cambridge, U.K.: Cambridge Univ. Press, 2009.

[25] T. Michaeli and Y. Eldar, "Minimum MSE estimation with convex constraints," in Proc. IEEE Int. Conf. Acoust., Speech, Signal Process. (ICASSP), 2007, vol. 3, pp. III-1093-III-1096.

[26] P. Gupta and P. R. Kumar, "Towards an information theory of large networks: An achievable rate region," IEEE Trans. Inf. Theory, vol. 49, pp. 1877-1894, Aug. 2003.

[27] P. S. Elamvazhuthi, P. S. Kulkarni, and B. K. Dey, "Improved layering protocol for wireless cooperative networks," in Proc. 2nd Int. Conf. Future Comput. Commun. (ICFCC), May 2010, vol. 2, pp. 333-337.

[28] A. Hjorungnes and D. Gesbert, "Complex-valued matrix differentiation: Techniques and key results," IEEE Trans. Signal Process., vol. 55, pp. 2740-2746, June 2007.

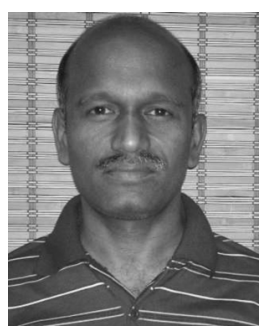

Pannir Selvam Elamvazhuthi (S'13) served in the Indian Navy from 1987 to 2009 after completing the B.Sc. degree in mathematics from Periyar Arts College, Cuddalore and the B.Tech. degree in electronics from the Madras Institute of Technology, Chennai, India. He received the M.Tech. degree in communication and radar engineering from the Indian Institute of Technology, Delhi, in 1992. During his tenure in the Indian Navy, he was deputed to Defense Research for eight years and served at the Naval Physical and Oceanographic Laboratory, Kochi and the Defense Institute of Advanced Technology, Pune, India.

From 2009 to 2012, he served in the Concept Lab of Innovation Group in Cognizant Technology Solutions, wherein he had collaborated with the Indian Institute of Technology Madras for executing technology projects. Since 2012, 
he has been serving at the Technical Center India, Delphi Automotive Systems at Bengaluru, India, as Engineering Group Manager, while also pursuing the $\mathrm{Ph}$.D. degree from the Department of Electrical Engineering, Indian Institute of Technology Bombay, Mumbai. His research interests are in wireless communication and radar/sonar signal processing.

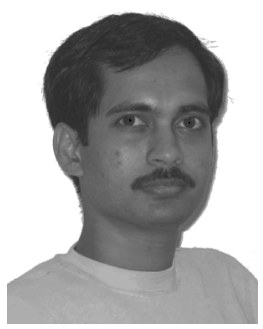

Bikash Kumar Dey (S'00-M'04) received the B.E. degree in electronics and telecommunication engineering from Bengal Engineering College, Howrah, India, in 1996. He received the M.E. degree in signal processing and the Ph.D. degree in electrical communication engineering from the Indian Institute of Science (IISc) in 1999 and 2003, respectively.

From August 1996 to June 1997, he worked at Wipro Infotech Global R\&D. In February 2003, he joined Hellosoft India Pvt. Ltd. as a Technical Member. In June 2003, he joined the International Institute of Information Technology, Hyderabad, India, as Assistant Professor In May 2005, he joined the Department of Electrical Engineering of Indian Institute of Technology Bombay where he works as Associate Professor. His research interests include information theory, coding theory, and wireless communication.
Dr. Dey was awarded the Prof. I. S. N. Murthy Medal from the IISc as the best M.E. student in the Department of Electrical Communication Engineering and Electrical Engineering for 1998-1999 and Alumni Medal for the best Ph.D. thesis in the Division of Electrical Sciences for 2003-2004.

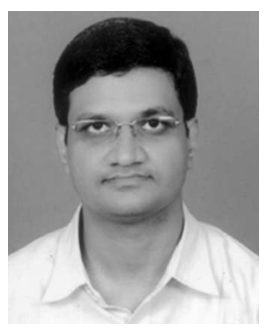

Srikrishna Bhashyam (S'96-M'02-SM'08) received the B.Tech. degree in electronics and communication engineering from the Indian Institute of Technology, Madras, in 1996 and the M.S. and Ph.D. degrees in electrical and computer engineering from Rice University, Houston, TX, in 1998 and 2001, respectively.

He worked as a Senior Engineer at Qualcomm, Inc., Campbell, CA, from June 2001 to March 2003 on wideband code-division multiple access (WCDMA) modem design. Since May 2003, he has been with the Indian Institute of Technology, Madras. He is now an Associate Professor in the Department of Electrical Engineering. His research interests are in communication and information theory, wireless networks, and statistical signal processing. 